\title{
IceCube: Performance, Status, and Future
}

Carsten Rott ${ }^{\mathrm{a}}$ for the IceCube Collaboration*

http://icecube.wisc.edu/science/publications/isvhecri2006.html

a Physics Department, Pennsylvania State University, 104 Davey Lab, University Park, PA 16802-6300, USA

\begin{abstract}
High-energy neutrinos are uniquely suited to study a large variety of physics as they traverse the universe almost untouched, in contrast to conventional astronomical messengers like photons or cosmic rays which are limited by interactions with radiation and matter at high energies or deflected by ambient magnetic fields. Located at the South Pole, IceCube combined with its predecessor AMANDA comprise the world's largest neutrino telescope. IceCube currently consists of nine strings, each containing 60 digital optical modules, deployed at depths of 1.5 to $2.5 \mathrm{~km}$ in the ice and an array of 16 surface air-shower stations. IceCube is expected to be completed in early 2011 at which time it will instrument a volume of one $\mathrm{km}^{3}$ below the IceTop air-shower array covering an area of one $\mathrm{km}^{2}$. The current IceCube detector performance is described and an outlook given into the large variety of physics that it can address, with an emphasis on the search for ultra-high-energy neutrinos which may shed light on the origins of the highest energy cosmic rays.
\end{abstract}

\section{Introduction}

Neutrinos open up a new window to the cosmos as they propagate undisturbed through the universe and therefore allow an undistorted view in contrast to messengers like photons or protons. Electrically charged particles bend in magnetic fields, therefore for protons below $10 \mathrm{EeV}$ $\left(10 \times 10^{18} \mathrm{eV}\right)$ any directionality information is distorted. Furthermore, above $50 \mathrm{EeV}$ protons interact with the cosmic microwave background radiation (CMB). This is known as the GreisenZatsepin-Kuzmin (GZK) cut-off [1. Neutrinos, on the other hand, allow one to study the entire energy spectrum.

\section{IceCube}

IceCube will instrument a volume of approximately one cubic kilometer with at least 70 strings arranged in a hexagonal pattern of $125 \mathrm{~m}$ string spacing. Each string contains 60 Digital Optical Modules (DOM) spaced evenly between a depth of $1450 \mathrm{~m}$ to $2450 \mathrm{~m}$. Each DOM consists of a $32.5 \mathrm{~cm}$ diameter pressure sphere that holds a downward facing 10" diameter HAMAMATSU

*http://icecube.wisc.edu
R7081-02 photomultiplier tube (PMT) with ten dynodes supported by coupling gel, a signal processing electronics board, an LED flasher board for calibration, and a high voltage base which powers the PMT at a gain of $10^{7}$. Signals above a threshold of 0.2 photo electrons (pe) are digitized in the ice by both a Fast Analog-to-Digital Converter (FADC) that samples at a fixed rate of $40 \mathrm{MHz}$ for $6.4 \mu \mathrm{s}$ and an Advanced Transient Waveform Digitizer (ATWD) sampling 128 bins of $3.3 \mathrm{~ns}$ width. To extend the dynamic range, the ATWD contains three channels with gains of $0.25,2$, and 16 . IceCube sensors have a substantially lower noise rate, while having an optical sensitivity that is about 1.4 times higher compared to its AMANDA counterparts. The dark noise rates of the DOMs in ice (with after-pulse suppression of $51 \mu \mathrm{s})$ is about $700 \mathrm{~Hz}(350 \mathrm{~Hz})$. This rate is further reduced to about 10 to $20 \mathrm{~Hz}$ by a local coincidence logic which requires activity in at least one of the adjacent DOMs in a $2 \mu \mathrm{s}$ time window. Above each IceCube string there is one IceTop surface station that consists of two tanks, each holding a low $\left(5 \times 10^{5}\right)$ and a high gain $\left(5 \times 10^{6}\right)$ DOM. This IceTop air shower array [2] is an integral part to the IceCube detector 
and relies on the same technology.

Before DOMs are deployed, they have to pass a rigorous test procedure. In addition, for a few DOMs a complete study of the absolute quantum efficiency and collection efficiency variations over the surface was conducted [3], to help determine systematic uncertainties related to the DOM surface efficiency variations, which was a limiting factor in many AMANDA analyses.

\section{Construction}

The IceCube detector is constructed at the South Pole during a short construction period lasting from early December to late January. During the first season 2004-2005 one string and four IceTop stations were installed, followed by eight more strings and 12 IceTop stations in 20052006. String deployment proceeds in less than 12 hours, into the $60 \mathrm{~cm}$ diameter holes, previously melted by an enhanced hot water drill to a depth of $2500 \mathrm{~m}$ in less than 35 hours. Information about ice properties was gathered through an in situ measurement with a system called the dust logger, which is lowered into the drill hole. This device sends out $410 \mathrm{~nm}$ laser pulses at $120 \mathrm{~Hz}$. Brushes, blocking the drill hole, ensure that light has to propagate through the ice, before being collected at the bottom of the system. In this way, the scattering and absorption coefficient have been determined with very high precision [4].

\section{Detector Performance}

Beginning with the deployment of the first string on January 28, 2005, IceCube's performance has been studied intensively. The first year single string data were found to be consistent with expectations [5]. IceCube's current multistring configuration allows for a more complex performance evaluation, which is the focus of this review.

The detector performance can be evaluated using data itself, complemented with simulation studies to understand uncertainties in the analyses better. Down-going muons produced naturally in cosmic ray showers transverse the detector and thus provide an ideal sample to study the detector. In addition, IceCube has artificial in situ light sources - 12 separately flashable LEDs for each DOM - that can be seen by adjacent DOMs and are ideal for studying a variety of detector properties.

The down-going muon sample is used to test the track reconstruction algorithms and also to study detector performance. They are ideal for long term stability studies, as they are continuously present in the physics data stream. Muon tracks are reconstructed with the standard IceCube offline reconstruction software that uses a $\log$ likelihood fit. Full waveform based reconstruction algorithms are currently under development.

\subsection{Timing}

Any physics analysis in IceCube relies on the precise knowledge of the relative timing of all the DOMs throughout the whole detector. We studied the timing variations of individual modules and the consistency of the timing of DOMs with respect to each other and found them to be consistent with design requirements.

\subsubsection{Timing Resolution}

The timing resolution of the DOMs has been studied in flasher data. For this, we determined the time difference between a flashing DOM and the time of the first hit on the DOM above it. The variation in the earliest hit time is related to the ice properties and the intrinsic timing resolution of the observing DOM. It is found to be better than 2 ns to 3 ns (see Figure 1). This result was also verified with several independent methods, using calibration signals (Rapcal) [5], flashers, and down-going muon data.

\subsubsection{Timing Consistency}

To check for consistency of the timing of an individual DOM with respect to the entire detector, we calculate the time residual, defined as the difference between the expected Cherenkov light arrival time from a reconstructed track and the observed hit time in a given DOM. The DOM under study is removed from the fit to avoid biasing the reconstruction.

The time residual distribution (see Figure 2) is expected to be peaked at zero if the timing of the 

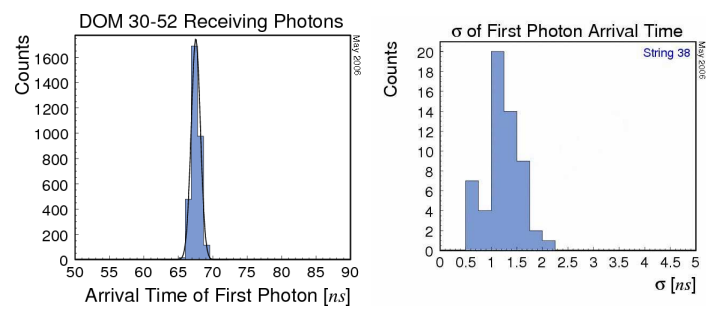

Figure 1. Left: The earliest hit time distributions from flasher data along with the fit to a Gaussian for a representative DOM. Right: The variation in hit time for the DOMs on a string.

module is consistent with the detector or otherwise shifted. The positive tail in the time residual distribution is from light that scatters before arriving at the sensor. Figure 3 shows the obtained prompt peak positions of the individual DOMs. The distribution is consistent with zero, likewise the spread arising predominantly from uncertainties in track reconstruction is within expectations.

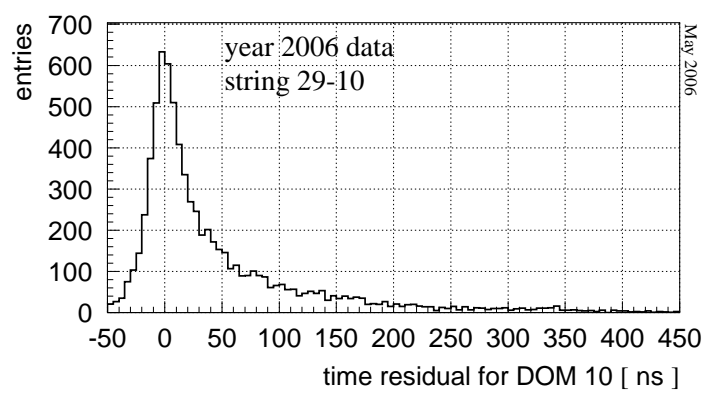

Figure 2. Time residual distribution from fits to down-going muons for a typical DOM.

\subsubsection{Long-term Timing Stability}

To verify long-term timing stability within a string, the hit time difference between adjacent

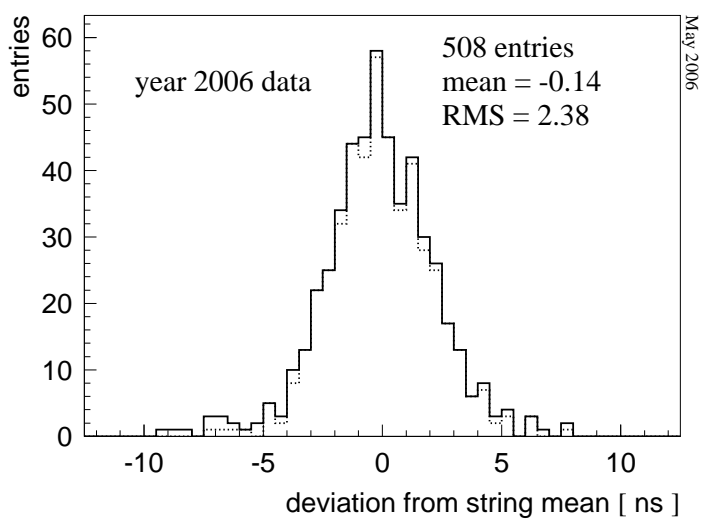

Figure 3. Deviation of the prompt peak position of the time residual distributions for "in ice" DOMs from the string mean.

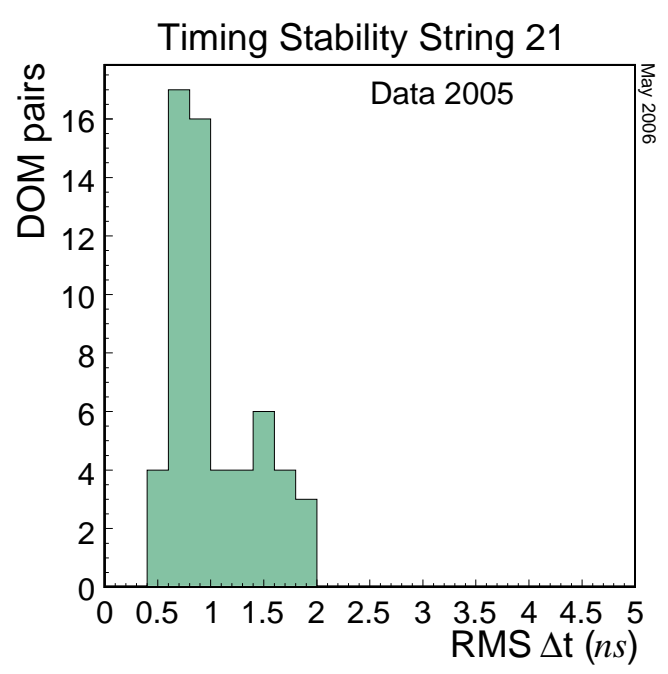

Figure 4. Variation of time difference between adjacent DOMs for selected, nearly vertical downgoing muon events over the course of 2005 . 
DOMs in events with a down-going muon running nearly parallel along the string were selected. The mean hit time difference was determined as a function of time. Figure 4 shows the obtained variation between adjacent DOMs over the course of a year. It was determined to be smaller than 2 ns, which was also confirmed by determining the variation in the prompt peak position of the time residuals over the year.

\subsection{Geometry Verification}

The detector geometry was initially determined through a laser range and depth sensor measurements at the time of deployment. The geometry within a string was verified by comparing hit times of adjacent DOMs from down-going muons and from flashers. The position was verified within about a meter - consistent with the expected error in the method. Relative depth of the strings with respect to each other and their distances in the ice were also verified using flasher data. In this method, the arrival time of unscattered light from a flashing DOM was determined for observing DOMs on a neighboring string by fitting the leading edge of the photon arrival time distribution. Using the speed of light in ice, the distance between the flashing DOM and each of the observing DOMs was obtained from the measured light travel time of earliest arriving photons. Results were obtained using different string pairs. With this method the geometry information can be verified within $1.0 \mathrm{~m}$.

\subsection{DOM Occupancy and Efficiency}

The occupancy for each DOM was determined in the down-going muon sample and the resulting distribution normalized by the total number of events for the string that satisfied the selection conditions (see Figure [5). The occupancy distributions of the various strings are consistent with each other and the observed structure is correlated with the optical properties of the ice as measured independently [4.

The efficiency of each DOM in the ice was studied by using reconstructed down-going muon tracks to determine the probability that a module observed a hit when a track passed within a given distance to it. This efficiency is found to be

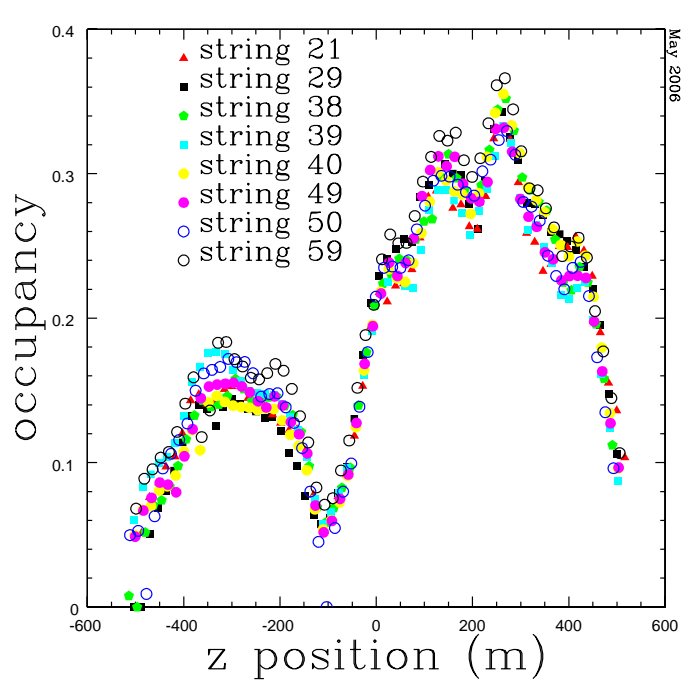

Figure 5. The DOM occupancy in the different strings are consistent with each other and features are clearly correlated with measured ice properties. The expected decrease in muon flux with depth is also observable $(\mathrm{z}=0$ marks the detector center).

consistent within the strings as shown in Figure 6 .

\subsection{IceTop - InIce Timing}

The timing between the IceTop air shower array and the in ice detector was verified by studying the time and spatial difference between hit modules of the two subdetectors. Figure 7 shows the obtained result, that is consistent with the speed of light as expected.

\subsection{AMANDA - IceCube Coincidence}

With its smaller OM spacings and interstring distances, AMANDA provides a compact subdetector array within IceCube. It is especially interesting for low energy analyses, like searches for WIMPs or slow moving particles. The joint operation of AMANDA and IceCube was tested by flashing DOMs in IceCube and observing the light in AMANDA and by extrapolating muon tracks from one detector into the other. Both 


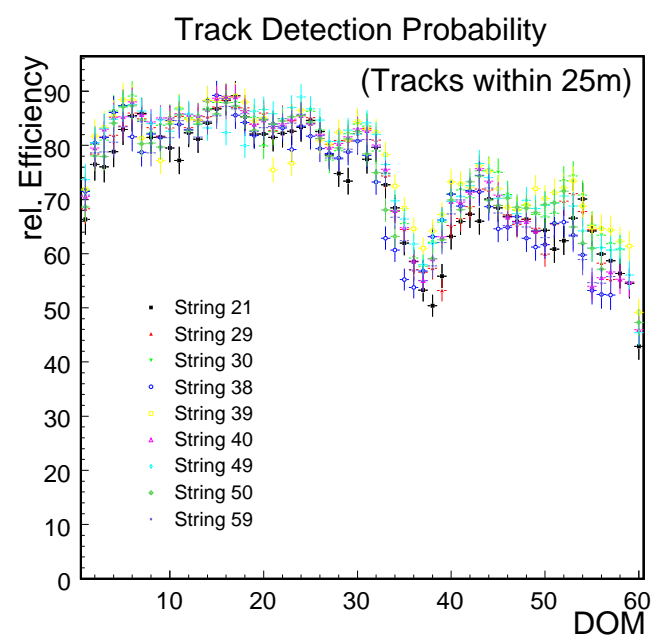

Figure 6. Probability of the DOMs in the ice to observe a hit if a reconstructed down-going muon passed within a given distance.

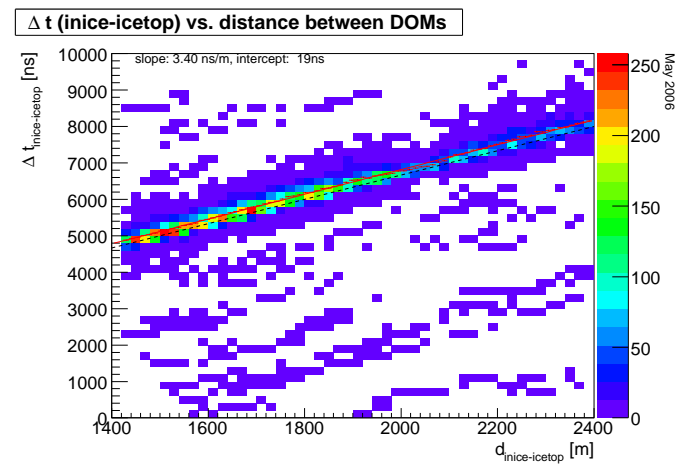

Figure 7. In-ice and IceTop timing is consistent. approaches confirm that AMANDA and IceCube can be jointly used and the first combined analyses are underway.

\section{Physics outlook}

IceCube can address a large variety of physics: Searches for weakly interacting massive particles (WIMPS), point sources, diffuse neutrino fluxes, transient sources, supernovae, GRBs, etc. IceCube may also observe spectacular signatures from tau neutrinos which would be very likely of extraterrestrial origin. IceCube's design has been optimized for $\mathrm{TeV}$ to $\mathrm{PeV}$ neutrino energies but can also be used to search for neutrinos at higher energies, even though such events might not be fully contained within the detector.

\subsection{Tau Neutrino}

The search for tau neutrinos is especially interesting as they are almost certainly of extraterrestrial origin and exhibit very distinctive signatures. Among those signatures is the double bang [6]: Two cascades are produced inside the detector volume as a result of a charge current interaction of an incoming $\nu_{\tau}$ and the sequential decay of the produced tau. At tau neutrino energies $E_{\nu_{\tau}}$ between $10^{15}$ and $10^{16} \mathrm{eV}$, the cascades are separated enough so that this signature can be observed with IceCube. At higher energies, the lifetime of the tau becomes large and it is likely that events are not fully contained within the detector volume, i.e. only a single cascade and the corresponding tau track would be observed. This type of event is referred to as a lollipop [7. Another class of events labeled sugardaddy 8, where the tau decays into a muon resulting in dim tau track followed by a brighter muon track, are also studied. The uniqueness of the tau signatures, the fact that they are almost certainly of extra-terrestrial origin and that these signatures have not been observed, makes these events especially interesting.

\subsection{Extremely High Energy Neutrinos}

Extremely energetic protons will lose their energy via a resonant scattering process with a photon in the $2.7 \mathrm{~K}$ CMB radiation, this is the GZK cut-off: $p+\nu_{2.7 k} \rightarrow \Delta^{*} \rightarrow N+\pi$. GZK neu- 
trinos are produced through the decay of the pions. The detection of these GZK neutrinos, of which IceCube is expected to observe one event per year 9], would supply firm evidence that the EHE cosmic rays are coming from extragalactic space. Through the study of the neutrino spectrum at GZK energies, we are also sensitive to annihilation or collapse of topological defects, that could generate EHE neutrinos with energies beyond the GZK scale.

\section{Future}

To obtain an EHE spectrum and perform point source analyses, a large statistical sample of EHE events is needed. In order to get a sufficient event rate, a detector of about 100 cubic $\mathrm{km}$ would be needed. The large absorption length for sound and radiowaves in the ice would make it feasible to instrument such a volume. Cherenkov radio signals have an attenuation length of about one kilometer. Radio is a technology that has been successful applied in RICE. Acoustic signals have an even larger attenuation length of the order of $10 \mathrm{~km}$, and the ice provides a potentially very quiet environment compared to for example the ocean. Hybrid neutrino detectors involving acoustics and radio in addition to optics can combine the individual properties of those detection methods and provide a broader and more robust detection of neutrinos in a cost effective way, while it also allows for cross-calibration of the individual detector components for improved systematics. Several technology options are currently actively explored.

For the season 2006-2007, prototypes for radio and acoustic sensors will be deployed along a few IceCube strings to study their performance.The South Pole Acoustic Test Setup (SPATS) [10] sensors, which consists of three channels (3 piezo ceramics, spaced at $120^{\circ}$ to allow for directional sensitivity) with low noise amplifier boards, will be deployed in three drill holes near the surface. The Askaryan Underice Radio Array (AURA) 11 system consists of sets of 4 sensors and one transmitter for calibration purposes and test modules will be deployed along several strings in the deep ice in the 2006-2007 deployment season.
Radio and acoustic methods are most effective for detecting electromagnetic or hadronic showers. Detection of muon tracks by these techniques is much more difficult. Initial sensitivity studies for EeV neutrino detection with a hybrid extension to IceCube have been performed [12].

\section{Conclusions}

IceCube's second year multistring configuration has been studied with down-going muons and artificial light sources. The performance was found to be in good agreement with the design specifications. We demonstrated the physics readiness of the detector and are collecting high quality physics data. In the coming season, we plan to more than double IceCube's current size by deploying another 12-14 strings. First physics analyses have started and we expect results soon.

\section{REFERENCES}

1. K. Greisen, Phys. Rev. Lett. 16 (1966) 748; G. T. Zatsepin and V. A. Kuzmin, Zh. Eksp. Teor. Fiz. 4 (1966) 114 [JETP Letters 4 (1966) 78].

2. X. Bai [IceCube Collaboration] same proceedings.

3. H. Miyamoto [IceCube Collaboration], astro-ph/0509330 pages 68-71.

4. J. Geophys. Res. 111 (2006) D13203.

5. A. Achterberg et al. [IceCube Collaboration], Astropart. Phys. 26, 155 (2006).

6. J.G. Learned and S. Pakvasa, Astropart. Physics J. 3, p. 267 (1995).

7. J. F. Beacom et al., Phys. Rev. D 68, 093005 (2003) [Erratum-ibid. D 72, 019901 (2005)].

8. T. DeYoung, S. Razzaque, and D. F. Cowen, astro-ph/0608486.

9. A. Ishihara [IceCube Collaboration], CRIS 2006.

10. S. Boser et al., Int. J. Mod. Phys. A 21S1 (2006) 221.

11. D. Williams [AURA Collaboration], ARENA 2006, To be published in the Institute of Physics conference journal.

12. D. Besson et al. [IceCube Collaboration], Int. J. Mod. Phys. A 21S1, 259 (2006). 